Mots. Les langages du politique

\title{
« Non au GA(z de)CHIS(te) ! » : étude diachronique des slogans de manifestations anti-gaz de schiste
}

"Non au GA(z de)CHIS(te) !": a diachronic study of demonstration slogans against fracking

«Non au GA(z de)CHIS(te) !»: estudio diacrónico de los eslóganes en las manifestaciones anti-gas de esquito

\section{Laurence Vignes}

\section{OpenEdition}

\section{Journals}

Édition électronique

URL : https://journals.openedition.org/mots/24442

DOI : $10.4000 /$ mots. 24442

ISSN : 1960-6001

\section{Éditeur}

ENS Éditions

\section{Édition imprimée}

Date de publication : 5 mars 2019

Pagination : 87-106

ISBN : 979-10-362-0130-1

ISSN : 0243-6450

\section{Référence électronique}

Laurence Vignes, « «Non au GA(z de)CHIS(te)! » : étude diachronique des slogans de manifestations anti-gaz de schiste », Mots. Les langages du politique [En ligne], 119 | 2019, mis en ligne le 01 janvier 2022, consulté le 23 avril 2022. URL : http://journals.openedition.org/mots/24442 ; DOI : https:// doi.org/10.4000/mots. 24442 


\section{« Non au GA(z de)CHIS(te) ! " : étude diachronique des slogans de manifestations anti-gaz de schiste}

La manifestation, une forme d'expression du répertoire protestataire, vise à mettre en scène une revendication (Fillieule, Tartakowsky, 2014). Les personnes collectives comme les individus mobilisent alors des stratégies de présentation d'eux-mêmes, au sein desquelles les slogans constituent un élément essentiel. C'est à cette production discursive spécifique que nous prêtons attention dans cet article, à propos de la mobilisation contre l'exploitation du gaz de schiste qui s'est développée en France entre 2010 et 2016.

L'objectif de ce travail est de caractériser le discours des manifestants à travers l'étude des énoncés spécifiques que sont les slogans montrés et scandés lors de ces manifestations. En retenant le contexte de communication qu'est la manifestation, nous privilégions la dimension sociale, historique et politique de ces "énoncés manifestants», selon le terme de Yana Grinshpun (2013). L'étude s'appuie sur deux corpus d'énoncés collectés dans les deux manifestations les plus significatives de la lutte contre le gaz de schiste. La première s'est déroulée à Villeneuve-de-Berg, village ardéchois, le 26 février 2011, et la seconde à Barjac dans le Gard, cinq ans plus tard, le 28 février 2016. Ces deux dates bordent quantité d'autres manifestations de moindre importance, qui se sont tenues dans l'ensemble des régions françaises concernées par les "permis de recherche d'hydrocarbures non conventionnels». Nous les mettons en relation parce qu'elles se répondent, d'abord par leur importance du point de vue du nombre de participants et donc de leur visibilité médiatique, ensuite parce qu'elles précèdent les deux lois françaises sur les «hydrocarbures non conventionnels» : la loi Jacob du 13 juillet 2011, et la loi Hulot du 30 décembre 2017.

Considérant que chacun des deux ensembles de slogans constitue une production homogène du fait de l'unité spatio-temporelle des dispositifs d'énonciation, nous porterons notre attention sur les caractéristiques énonciatives d'une part, puis thématiques et lexicales d'autre part, de chacun de ces discours manifestants. Il s'agira également d'interroger la circulation de certains 
slogans qui, en détournant des énoncés antérieurs, convoquent des valeurs, des références témoignant du travail des énonciateurs pour se forger une identité. Cette dernière s'inscrit évidemment dans les mots eux-mêmes, mots qui sont lestés des sens dont les emplois antérieurs les ont chargés (Siblot, 1999).

\section{Slogans et particitation militante}

La définition du slogan du philosophe Olivier Reboul est devenue classique :

J'appelle slogan une formule concise et frappante, facilement repérable, polémique et le plus souvent anonyme, destinée à faire agir les masses tant par son style que par l'élément d'auto-justification, passionnelle ou rationnelle, qu'elle comporte : comme le pouvoir d'incitation du slogan excède toujours son sens explicite, le terme est plus ou moins péjoratif. (Reboul, 1975, p.42)

Cet ensemble de traits concerne partiellement notre objet d'étude : si les caractères polémique et pragmatique sont indiscutables, il n'en va pas de même pour la concision et l'anonymat. En effet, nous avons collecté l'ensemble des énoncés produits et montrés, sinon brandis, dans le cadre d'une même situation de communication délimitée par le temps de la manifestation. Les ouvrages traitant du slogan analysent quant à eux des unités sélectionnées différemment, dans une optique rhétorique et centrée sur la fonction poétique (voir Grunig, 1990). Nous avons donc opté pour le cadre proposé par Dominique Maingueneau (2012, 2004), qui offre une approche énonciative mieux adaptée à notre projet. Pour cet auteur, les slogans politiques sont des énoncés relevant par nature de la «particitation ${ }^{1}$ militante», permettant de «renforcer la cohésion d'une collectivité en l’opposant à un extérieur menaçant». Produits par un énonciateur collectif, les slogans ont vocation à être répétés, à l'infini lorsqu'ils sont scandés, ou bien d'une particitation (affiche, tract, manifestation) à l'autre. La collectivité qui s'exprime alors en «nous» forme un groupe «cimenté par une finalité commune » (Maingueneau, 2004, p. 119), laquelle peut être transitoire. Ce qui suit présente bien les mécanismes que nous nous proposons d'étudier :

Lors d'une manifestation qui rassemble une population hétérogène autour d'une question d'actualité, le slogan n'a pas d'autre communauté support que le groupe lui-même qui est en train de l'énoncer; de là une tendance à fragmenter les slogans en fonction des sous-groupes qui composent cette communauté transitoire. La communauté transitoire fabrique un Thésaurus conjoncturel (les slogans co-présents dans l'espace-temps de cette manifestation), qui mêle des slogans de circonstance et d'autres qui reviennent d'une manifestation à l'autre.

1. Ce mot valise «qui mêle participation et citation » désigne des énoncés détachés par nature ou fonction, mémorisables et autonomes. 
[...] Il y a néanmoins particitation, car les divers slogans impliquent la place d'un hyperénonciateur dont l'autorité fonde l'ensemble des slogans compatibles dans l'espace de la manifestation : cette entité [...] doit exister au-delà de ce rassemblement fugace, assurer une continuité imaginaire d'un rassemblement à l'autre. (Maingueneau, 2004, p.120)

Cette continuité est d'autant plus forte dans le cas de la lutte contre le gaz de schiste, qu'elle s'étale sur plusieurs années (entre 2011 et 2018), et se répartit entre un grand nombre de collectifs regroupant, dans toutes les régions concernées, des citoyens et des élus. Nos corpus de slogans émanent d'énonciateurs collectifs produisant des "Thésaurus conjoncturels», dont les composants deviennent plus ou moins pérennes, en fonction de facteurs que nous examinerons attentivement.

\section{Composition des corpus}

Ils ont été constitués à partir de photos et surtout de vidéos d'amateurs, disponibles sur le site YouTube. Les vidéos sont précieuses car elles permettent l'accès aux éléments sonores - prises de parole officielles, slogans scandés comme aux éléments visuels, les slogans écrits. Comme le constate Alexandre Lambelet (2010) : «L'enregistrement vidéo ou photographique permet de détacher le moment de l'observation du déroulement de la situation observée, et cette distance rend possible une exploration répétée du document, ainsi que la découverte de nouvelles caractéristiques autrement inaperçues. » En jouant de l'arrêt sur image, nous avons pu collecter 100 slogans dans 14 vidéos (65 min de film) consacrées à la manifestation de Villeneuve-de-Berg, et 112 slogans (86 min de film) pour celle de Barjac. La multiplicité des films traitant du même événement présente un autre avantage : en prospectant l'ensemble des vidéos accessibles, nous avons pu compléter régulièrement nos relevés pour tendre vers la saturation. Le corpus ainsi collecté constitue un ensemble fiable et représentatif.

\section{Circulation des slogans entre modes sémiotiques}

Ce mode de recueil nous a permis de recueillir 15 slogans scandés, soit $7 \%$ des deux corpus. Les vidéastes amateurs auraient-ils négligé ce composant de l'événement? Nous pensons plutôt que d'autres éléments ont suppléé à leur recours. En effet, au sein de l'ensemble des signes de reconnaissance et d'identification constituant la scénographie construite par les manifestants (Fillieule, Tartakowsky, 2014, p. 167), le slogan scandé n'est qu'un élément parmi d'autres. Dans les manifestations étudiées, nous avons noté l'importance 
donnée aux prises de parole des élus et aux chansons, créations originales ou reprises. L'une d'elles, un pastiche de La Montagne de Jean Ferrat², se signale par sa récurrence. En quelques images, la situation est décrite :

Ils veulent rester dans ce pays

Pour travailler gagner leur vie

Qu'ils soient venus qu'ils y soient nés

Le gaz tranquille sous la terre

Dormait depuis des millénaires

Sans jamais faire chier personne

Voilà qu'une bande de milliardaires

Viendrait ici tout foutre en l'air [...]

La chanson appartient au «matériel révolutionnaire» (Calvet, 1976), et contribue à la construction de l'identité du groupe d'opposants de manière d'autant plus efficace que la mélodie facilite la diffusion du texte. Ce pastiche de La Montagne participe pleinement au Thésaurus de la communauté manifestante, et illustre fort bien l'opposition entre les deux groupes. Nous avons cependant choisi de limiter notre étude aux unités de taille plus restreinte que sont les slogans3. On observe en effet une circulation entre slogans écrits et oraux. Les quelques slogans scandés que nous avons relevés sont repris sur des panonceaux, tel le célèbre «nogazaran» (voir infra). Les slogans écrits constituent donc la majeure partie (93\%) du corpus. Au sein de ces items écrits, certains slogans présentent des caractéristiques rythmiques, propres au slogan scandé, qui font qu'à la lecture, ils sonnent. Comme le constate Louis-Jean Calvet : «je sais en entendant un syntagme, s'il s'agit d'un slogan ou d'une phrase “normale”, je le sais même en le lisant » (1976, p. 80). Les compétences pulsionnelle, rythmique et linguistique des locuteurs se combinant dans l'activité de réception (et de production) de slogans leur permettent cette reconnaissance. D'autres énoncés, nous le verrons, ne possèdent pas les qualités rythmiques permettant la circulation d'un mode sémiotique à l'autre.

\section{Hyperénonciateur et communauté transitoire}

Se pose alors la question de la production des slogans. Nous avons convoqué la notion d'hyperénonciateur4 (Maingueneau, 2004) afin de pouvoir constituer, puis examiner globalement les productions de chacune des manifestations

2. Jean Ferrat a écrit La Montagne en 1964, à Antraigues-sur-Volane, village ardéchois où, définitivement installé par la suite, il est considéré comme l'enfant du pays.

3. Le refrain de la chanson se retrouve dans plusieurs slogans de la manifestation de 2011.

4. L'hyperénonciateur apparaît comme une instance qui, d'une part, garantit l'unité et la validité de l'irréductible multiplicité des énoncés du Thésaurus, et d'autre part confirme les membres de la communauté dans leur identité, par le simple fait qu'ils entretiennent une relation privilégiée avec lui. 
observées. Comme l'écrit cet auteur (voir supra), des sous-groupes composent cette communauté transitoire, ce qui a pour conséquence la fragmentation des slogans. Dans le corpus qui nous occupe, certains éléments permettent leur identification. Il s'agit en premier lieu de la dimension matérielle du support de slogan, dimension constitutive, comme le rappelle D. Maingueneau : «le médium n'est pas un simple “moyen”, un instrument pour transporter un message stable : une transformation importante du médium modifie l'ensemble d'un genre de discours5 » (2016, p. 69).

Nous distinguons deux types de médiums, en fonction de l'énonciateur du slogan. Nous regroupons, d'une part, quelle que soit leur taille, les supports matériels émanant de collectifs, depuis l'affichette jusqu'à la bannière. Ils se distinguent par une écriture typographique, des logos ou illustrations imprimés et une signature indiquant leur origine. Ils sont le produit d'un circuit communicationnel, d'une complexité impossible à détailler ici. Les slogans de collectifs sont arborés dans chaque manifestation par les élus en écharpe, qui, en soutenant ainsi - au double sens du terme - le discours des opposants, renforcent la légitimité de cet hyperénonciateur. Nous qualifions les slogans brandis sur ce type de supports d'officiels.

Cependant, la grande majorité des productions émane des manifestants eux-mêmes, comme le montre le médium, souvent en carton brut ou papier. La plus grande variété est alors observable, depuis le panonceau soigné, jusqu'au slogan griffonné sur une plaque de carton, porté sur le sac à dos ou à même le corps, qui devient alors le support essentiel d'un signe à vocation identitaire (Memmi, 1998). Des objets, parmi lesquels est privilégié le masque à gaz, participent à la mise en scène. Une fourche en bois noueux portant l'inscription "Sarko y’a pas moyen dégage » illustre le discours de son auteur, qui suggère ainsi que son outil agricole pourrait bien se transformer en arme.

Ces énoncés individuels ne répondent pas obligatoirement aux caractéristiques constitutives du genre du slogan. Certains d'entre eux montrent des qualités formelles, que ce soit sur le plan sonore («n'inschistez pas on est pas des kamigaz» / "l'eau c'est la vie défendons-la sans parti-pris») ou visuel («Non au Gaz(s)chis(te)» / «\$chi\$te!»). Brefs, efficaces, denses, ces slogans révèlent un certain talent.

D’autres énoncés expriment plus prosaïquement le message de leur auteur : «Où qe ce soit dans tous les pays du monde : non au gaz de schiste non à Total et à tous ses produits carburants uiles, gaz...! Non à tous les lobbis qels qu'ils soient! » (sic). Brandi fièrement par un vieux monsieur souriant, ce message est un énoncé manifestant à part entière, qui fonctionne comme slogan au sens élargi, par son énonciation et son support. Nous qualifions ces slogans, émanant sinon d'individus, de subjectivités assumées comme telles, d'artisanaux.

5. L'auteur souligne. 
Officiels et artisanaux, ces deux types de slogans constituent l'ensemble du discours manifestant pris en charge par un hyperénonciateur «opposant au gaz de schiste».

\section{Contextualisation : des débuts à Villeneuve-de-Berg}

Au printemps 2010, le ministre de l'Écologie, Jean-Louis Borloo, accorde à plusieurs sociétés pétrolières françaises et étrangères des permis de recherche "d'hydrocarbures liquides et gazeux». Les rares journaux qui en rendent compte pendant l'été le font sans tonalité critique. Le basculement se produit lorsque Fabrice Nicolino, journaliste indépendant, lance l'alerte en publiant le 6 octobre 2010 dans Charlie Hebdo un article intitulé «Gazarem lou Larzac ». Le célèbre plateau se retrouve en effet inclus dans la zone de $4414 \mathrm{~km}^{2}$ couverte par le permis d'exploration dit de «Nant». José Bové, député européen connu pour son engagement dans la défense du Larzac où il réside, va incarner ce nouveau combat. Le premier comité de vigilance se crée fin 2010. Puis, en un mois, la pétition sur internet «Gaz de schiste non merci! »6 recueille 50000 signatures, tandis que se développent les réunions d'information citoyenne, au cours desquelles le film Gasland est diffusé. Face à cette mobilisation «fulgurante» (Chateauraynaud, 2011), la nouvelle ministre de l’Écologie, Nathalie Kosciusko-Morizet7, annonce le 2 février la suspension des travaux d'exploration. Mais la contestation continue de s'étendre, et le 26 février se tient à Villeneuve-de-Berg le premier rassemblement national contre le gaz de schiste.

Les estimations oscillent entre 10000 et 20000 personnes, rassemblées autour de J. Bové; certains y voient un «front de refus extrêmement bigarré », selon Pierre-Marie Terral, l'historien du Larzac (2017), d'autres «une foule bon enfant» (Le Monde, 26/02/2011). Sont présents des citoyens, des membres de collectifs des départements concernés par les permis, de nombreux élus locaux et régionaux. On compte également des écologistes, des militants d'associations et de partis politiques. Le lieu où se tient la manifestation revêt une importance particulière, puisqu'elle se déroule non pas en milieu urbain, dans le lieu de contestation symbolique de la rue, mais sur une route, plus précisément sur une aire de repos, qui est aussi un belvédère ${ }^{8}$. Le paysage constitue alors un argument visuel d'une importance majeure, comme l'exprime le maire de Villeneuve-de-Berg :

[...] de ce belvédère, on a une si belle vue sur les Cévennes et à nos pieds, les territoires agricoles vastes et productifs notamment le domaine agricole du Pradel cher à Olivier de Serres. Et l'on voudrait, qu'au milieu de ce décor, on vienne installer des plates-formes de forage tous les 200 ou 300 mètres?

6. Cette pétition est encore accessible en ligne à l'adresse : https://www.petitions24.net/signatures/gaz_de_schiste_non_merci/start/128220 (consulté en mars 2018).

7. Elle succède à J.-L. Borloo en novembre 2010.

8. Voir figure 1. 


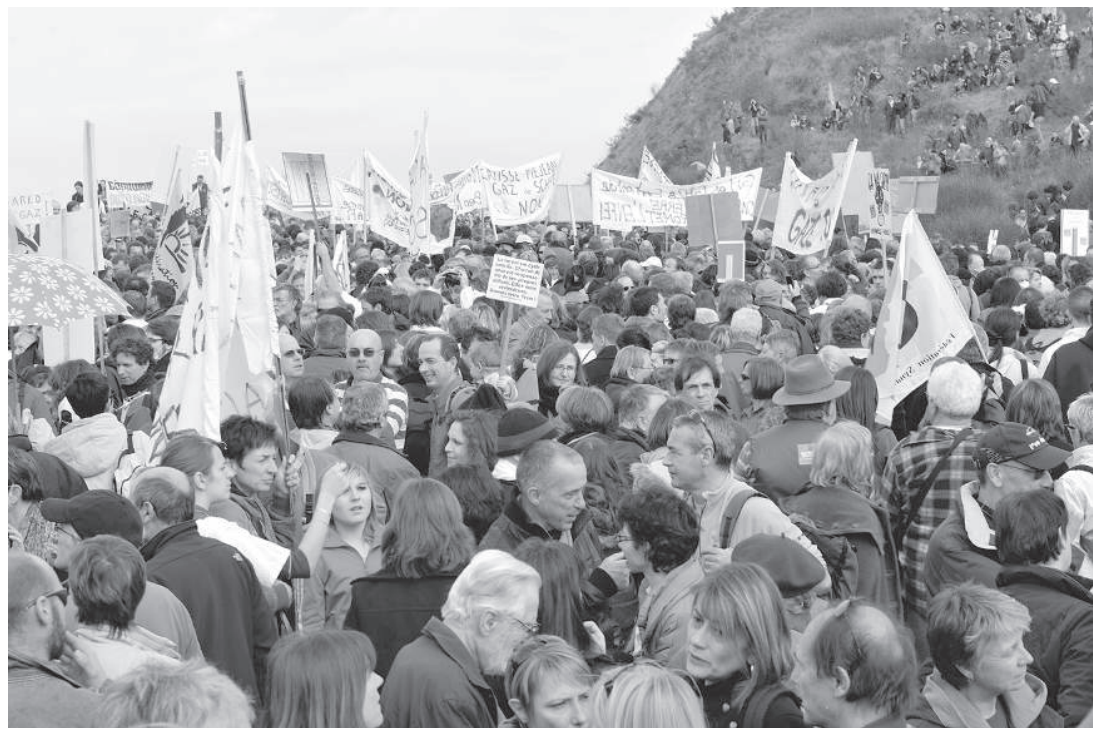

Figure 1. Le belvédère de Villeneuve-de-Berg, 26 février 2011

(c) Pascal Blachier

\section{Slogans officiels de Villeneuve-de-Berg}

Sur les 100 slogans de ce premier corpus, répertoriant des formes différentes et non des occurrences, impossibles à dénombrer, seuls 3 relèvent de l'énonciation de collectifs. Le caractère récent de la mobilisation explique peut-être ce fait. Le plus fréquent est le slogan du collectif o7 «Stop au gaz de schiste», accompagné du logo au masque à gaz, logo déposé et protégé dans lequel le o du mot «stop » est remplacé par la forme ovale d'un masque à gaz, renvoyant au syntagme «gaz de schiste » placé au-dessous. Signifier le « gaz » par l'objet masque à gaz permet d'abord la représentation, par métonymie, d'une matière invisible par définition et, culturellement, il renvoie à un symbole de la Première Guerre mondiale, le «masque MCG (Matériel Chimique de Guerre) ». Ce masque, doté d'une cartouche filtrante, véhicule un imaginaire de mort et de souffrance, en renvoyant aux soldats gazés au chlore ou à l'ypérite. Symbole puissamment empreint de négativité, le masque à gaz souligne efficacement le message des opposants : le gaz de schiste est dangereux, il faut s'y opposer ${ }^{10}$. D'autre part, l'objet active un second réseau de significations, plus contemporain : il évoque le recours des forces de l'ordre aux bombes lacrymogènes, lors

9. Voir figure 2.

10. Un slogan artisanal exprime cette idée : «Gaz de schiste : non au gaz de la mort». 
des manifestations sur les sites de ZAD ${ }^{11}$. Le symbole signale ainsi l'inscription dans l'histoire de ces luttes d'opposition aux « grands projets inutiles (et imposés) » selon l'expression utilisée au sujet du projet d'aéroport à Notre-Damedes-Landes ${ }^{12}$. Enfin, le dessin se prête bien à la reproduction grandeur nature ; nombre de manifestants portent ce masque en carton au-dessus d'une combinaison blanche. L'utilisation de ce symbole dans le déguisement en renforce la diffusion, et permet un détournement carnavalesque qui le dédramatise.

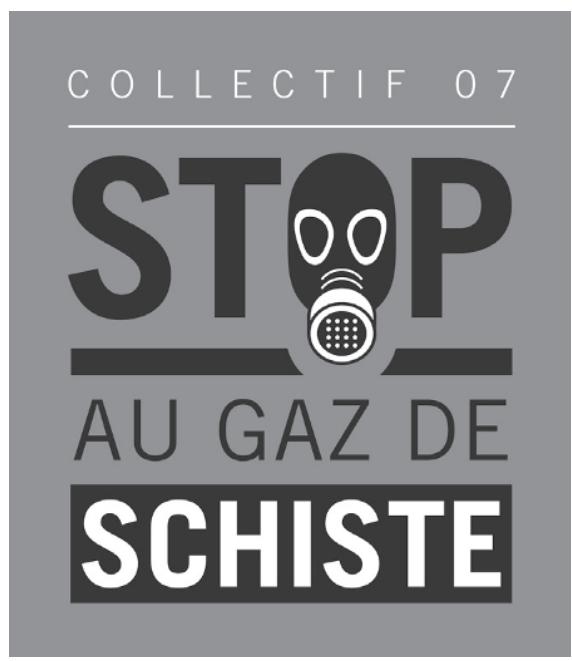

Figure 2. Logo du collectif o7 Stop au gaz de schiste en 2011

(c) Collectif 07 Stop au gaz de schiste

Le second slogan officiel est celui arboré par les élus en écharpe tricolore, portant en tête de cortège une longue bannière : "Élus solidaires - Non au gaz de schiste». Tout comme le «stop» du premier slogan, le «non», expression la plus commune du refus, déclinée sur nombre de panonceaux individuels, remplit la première condition de «la communication d’opposition » (Libaert, Pierlot, 2015, p. 37) : la nécessité de marquer clairement une opposition forte et résolue dès les premiers moments. Elle constitue la première caractéristique de l’hyperénonciateur de la manifestation.

11. Le sigle des zones à aménagement différé (ZAD) a été détourné par les militants contestant les projets pour désigner les zones à défendre. Les plus connues sont celles de Notre-Dame-desLandes, Sivens et Bure.

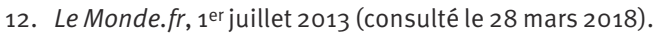




\section{Slogans polyphoniques}

Considérons à présent les slogans polyphoniques, traversés par des voix étrangères. Nous suivrons $D$. Maingueneau qui pose la question en termes de particitation et d'hyperénonciateur. Lorsque le participant à la manifestation lit (les slogans étant surtout écrits) le slogan polyphonique, il est supposé reconnaitre la citation, sans que le locuteur citant n'en ait indiqué la source, puisqu'il fait partie de la même communauté. Lorsque le lecteur n'en fait pas partie, le discours est reçu comme signe d'opposition qui invite à entrer dans la communauté d'opposants. La notion de Thésaurus se révèle alors particulièrement pertinente :

Le locuteur citant montre son adhésion à l'énoncé cité, qui appartient à ce que l'on pourrait appeler un Thésaurus d'énoncés, aux contours plus ou moins flous, indissociables d'une communauté où circulent ces énoncés et qui, précisément, se définit par le partage privilégié d'un tel Thésaurus. [...] Ce Thésaurus et la communauté correspondante sont référés à un hyperénonciateur dont l'autorité garantit moins la vérité de l'énoncé [...] mais plus largement sa "validité», son adéquation aux valeurs, aux fondements d'une collectivité. (Maingueneau, 2004, p.113)

Du point de vue formel, nous avons affaire à la figure du détournement (Leroy, 2005, p. 201), dont la «capacité à faire entendre, outre la voix de l'énonciateur, une (ou plusieurs) autre(s) voix, qui le feuillettent énonciativement» est typique du dialogisme. Rien ne garantit, cependant, que le lecteur non averti retrouve sous le détournement le dit initial. Son rôle reste décisif dans le fonctionnement du détournement.

\section{«Gaz de schiste : non merci!»}

Ce slogan, souvent inscrit dans un logo noir et blanc ${ }^{13}$ représentant une coupe de terrain avec un derrick en surface, a été choisi par plusieurs collectifs. Il réfère au slogan le plus célèbre de la lutte antinucléaire « Nucléaire? Non merci », traduction de l'original danois de 1975 : «Atomkraft? Nej tak». Cependant, dans le slogan détourné «Gaz de schiste : non merci!», le point d'interrogation qui fait du segment « Nucléaire? » du slogan initial une question, voire une hypothèse, a disparu. Reste un énoncé de refus poli mais ferme, le point d'exclamation final l'atteste. Le gaz de schiste et le nucléaire relèvent du même domaine de lutte environnementale, l'énergie, ce qui accentue la cohérence au sein du détournement. Cependant, rares sont les slogans qui évoquent le précédent nucléaire, dont la place dans le Thésaurus se limite à quelques énoncés artisanaux :

Nucléaire gaz de schiste démocratie bafouée

Areva Total même combat

13. Voir figure 3. 


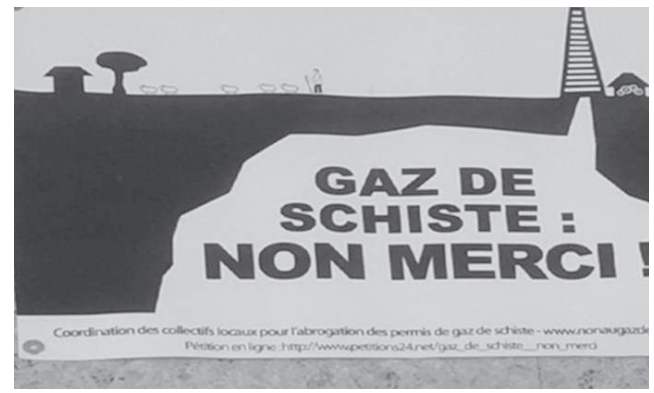

Figure 3. Logo de la Coordination nationale contre les gaz de schiste en 2011 (c) Coordination nationale des collectifs locaux CGHS (contre les gaz et huiles de schiste)

L'historienne du nucléaire Sezin Topçu, dans son ouvrage de 2013 intitulé La France nucléaire, analyse les stratégies gouvernementales ayant conduit au succès de la nucléarisation de la France, en dépit de fortes résistances citoyennes. Est-ce l'insuccès de ces luttes qui pourrait expliquer la faible place de ces citations dans le Thésaurus?

\section{«No gazaran»}

La lutte anti-gaz de schiste s'oriente vers une référence plus valorisante avec le célèbre slogan des républicains espagnols : «No pasarán »14. Le détournement s'opère par une modification formelle mineure portant sur deux phonèmes transformant «pasarán» en "gazaran». Le contexte sociohistorique ainsi que la forme "gaz» orientent aisément l'interprétation de ce poétique néologisme, dont l'équivalent espagnol serait "gasearán». Toutefois, ce dernier, plus éloigné formellement de l'unité « gaz», n'a pas été choisi. « No gazaran» reste certainement le slogan le plus repris dans la presse au moment de la mobilisation en 2011 et jusqu'en 2016. Il fait partie des rares slogans scandés, grâce à son rythme binaire, une des caractéristiques primordiales de ces énoncés selon L.-J. Calvet (1976). No gazaran est enfin le titre d'un documentaire de $2014^{15}$ qui a connu un certain succès.

Les trois occurrences de notre corpus permettent d'en préciser le sens :

Le fast schiste ne passera pas - No gazaran!

No Gazaran Total Devon Shuepbach Gdf Suez DEGAZEZ!

No gazaran lo Larzac

14. "Ils ne passeront pas», slogan des républicains en lutte contre les nationalistes fascistes du général Franco, en 1936.

15. Documentaire de Doris Buttignol et Carole Menduni, DVD, éditions Montparnasse, 2014. 


\section{No gazaran ou gardarem?}

La relation avec le slogan originel est attestée par le calembour « fast schiste», qui renvoie au slogan antérieur détourné : «le fascisme ne passera pas». L'allusion est plus discrète dans la seconde occurrence qui assigne la place de l'oppresseur aux pétroliers. Le dernier énoncé ajoute une captation (Maingueneau, 2016) avec la précision «lo Larzac», qui renvoie au slogan phare de la lutte contre l'extension du camp militaire (entre 1970 et 1981) : «Gardarem lo Lazac ». L'occitan, langue du slogan larzacien, est d'ailleurs présent dans quelques unités de notre corpus :

Total GDF Shuepbach passaran pas Gardarem l'ardécha sensa gaz de sistre

Dans ce slogan, la lutte antifasciste espagnole ( passaran pas $»^{16}$ ) et celle du Larzac («Gardarem ») se combinent explicitement. Ces rapprochements de signifiants traduisent, tout en la construisant, une parenté entre les causes défendues. Cette parenté est géographique (le Larzac), actancielle (J. Bové), linguistique (espagnol et occitan) et bien sûr factuelle, puisqu'il s'agit dans les deux cas de résister à un oppresseur. La mise en relation entre les deux luttes françaises avait déjà été tentée par F. Nicolino, dans le titre de son article d'alerte « Du gaz à tous les étages: Gazarem lou Larzac ». La forme * gazar, non attestée en occitan, que l'on retrouve dans «no gazaran » apparaissait déjà. La traduction donne toutefois un étrange résultat: «nous gazerons le Larzac ». Estce le propos d'un pétrolier? Dans ce cas, l'énoncé initial «Gardarem lo Larzac» ferait l'objet d'une parodie, ce que le contenu de l'article, incitant à l'opposition, dément. Il aurait été plus logique d'énoncer «ils gazeront»: «gazaran ». Peut-être le locuteur s'est-il laissé entrainer par la forme en «em »? Quoi qu'il en soit, le rapprochement entre Larzac et gaz de schiste est attesté hors corpus. Dans un article du Midi Libre du 20 janvier 2011 intitulé «Gaz de schiste : la nouvelle bataille du Larzac », J. Bové affirme : «Schuepbach Energy a eu tort de choisir le Larzac [...] s'ils venaient je pense qu'ils seraient “reçus". La lutte ici on connait». De même, dans la postface de son dernier ouvrage, Le Larzac terre de lutte : une contestation devenue référence, P.-M. Terral (2017, p.129) explique : «Par son issue victorieuse, par l'inventivité de ses actions inédites, humoristiques et festives, autant que par les valeurs dont il a été porteur, le Larzac demeure une lutte de référence, conservant une capacité d'inspiration pour les mouvements ultérieurs». Nous confirmons qu'elle s'inscrit linguistiquement dans le discours manifestant de la lutte contre le gaz de schiste.

16. Ici formulé en occitan. 


\section{Étude lexicale du corpus de Villeneuve-de-Berg}

Il s'agit à présent de considérer les arguments avancés dans les slogans, lesquels se répartissent en deux thématiques principales : la critique des politiques et l'expression des risques liés au gaz de schiste.

\section{Borloo ou Sarko?}

Les slogans visent majoritairement deux personnalités politiques : J.-L. Borloo, responsable de la délivrance des permis d'explorer, et Nicolas Sarkozy, président de la République. Aux slogans classiques, déclinables à l'infini sur l'axe paradigmatique (Calvet, 1976, p. 78), du type «Borloo démago / à l'échafaud / beau boulot», s'ajoutent des unités jouant sur le signifiant : «Borloo Saloo », «Non à l'environnement à la bord-l'eau ». Les rimes en « 0 » dominent, y compris en occitan : «Borloo Kosciusko De qué sa ko ces écolos?». Lorsqu'il est question de J.-L. Borloo, les slogans parlent essentiellement de lui, mais ne lui parlent pas, à l'exception de l'impératif «Borloo va forer chez ta mère», et du constat adressé "Borloo tu nous l'as fait dans l'dos». En revanche, lorsqu'il s'agit de N. Sarkozy, c'est l'inverse. Tandis que J.-L. Borloo n'est plus ministre au moment de la manifestation, N. Sarkozy candidate pour un second mandat. La proximité de l'élection présidentielle induit certainement les invites dont il fait l'objet, et qui lui sont directement adressées. Les cinq slogans récoltés lui intiment tous de «dégager», plus précisément de «dégazer». Il se produit un nouveau détournement, celui du slogan adressé en 2011 au président tunisien Ben Ali. Sur le plan formel, l'attractivité du morphème «gaz» se confirme avec la paire de paronymes «dégage/dégaze», à laquelle s'ajoute «cass(e)/ gaz(e) » dans l'énoncé détaché, fortement surasserté : «cass' toi pauv' con »17, retourné contre son énonciateur initial en «gaz' toi pauv' con». La subversion vise clairement à disqualifier l'énonciateur du propos capté.

\section{Le gaz de schiste : quelles représentations?}

Le second thème porté par les slogans, c'est l'objet topique, le gaz de schiste lui-même. Le syntagme "gaz de schiste » compte 19 occurrences pour 63 de la forme abrégée «gaz». En revanche, le syntagme caractéristique du discours des promoteurs, «gaz non conventionnel», est stigmatisé. Ses deux seules occurrences se situent dans des contextes dépréciatifs. Citons, pour exemple, le panonceau qui représente l'ancien ministre de l'Écologie, un volumineux

17. Cette réplique de Nicolas Sarkozy, en visite au salon de l'agriculture le 23 février 2008 , adressée à un militant qui refusait de lui serrer la main, a été abondamment reprise et médiatisée. 
dossier vert sous le bras, pantalon aux genoux, lâchant un vigoureux « prout », qui permet d’interpréter la légende : «le seul gaz non conventionnel acceptable!» Dans un des rares slogans dont l'image conditionne la compréhension, c'est une paire de fesses qui tient, si l'on peut dire, le même prosaïque propos $^{18}$.

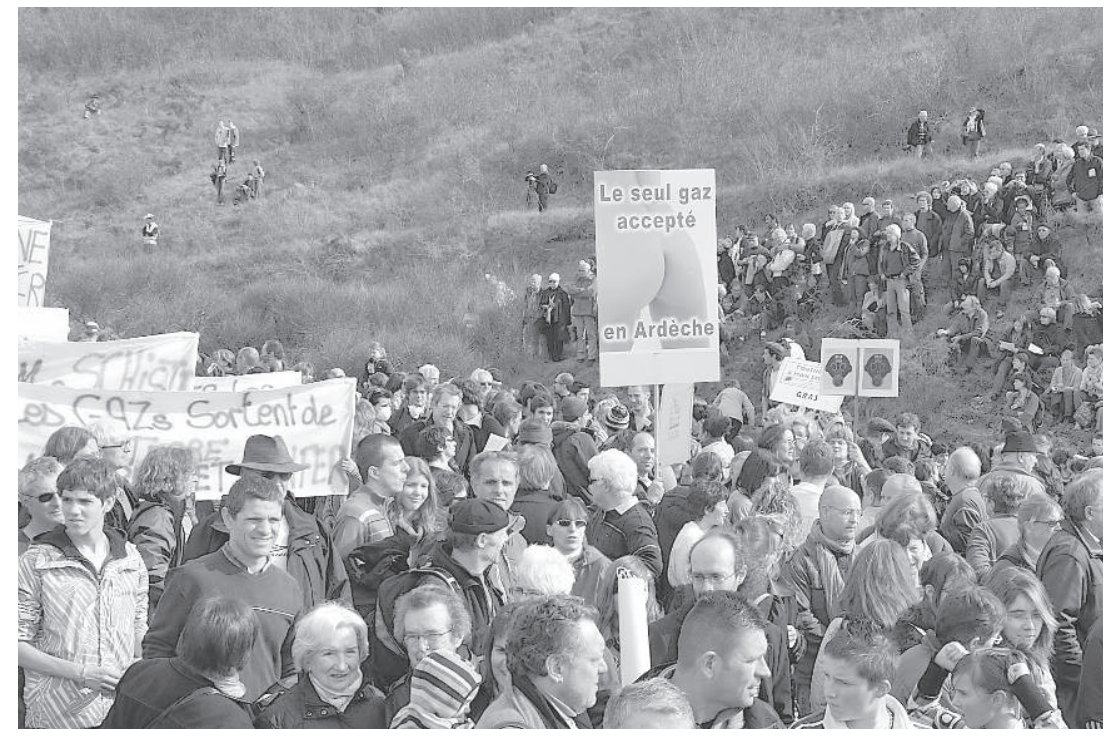

Figure 4. «Le seul gaz accepté en Ardèche », Villeneuve-de-Berg, 26 février 2011 (C) Pascal Blachier

Quels sont les traits retenus par le discours manifestant pour construire cet objet topique? Le premier d'entre eux concerne les compagnies pétrolières et leurs objectifs (12 items). Elles sont désignées nommément dans quatre slogans dans lesquels les détenteurs du permis de Villeneuve-de-Berg (Schuepbach et Total) sont cités. D’autres désignations s’observent («Pétroliers ${ }^{19}$ dehors!»), dont un néologisme : «Gazoschisteurs à la mémoire courte le Grenelle n'est pas si loin. Fuyez ». Dans sept slogans, l'objectiffinancier des pétroliers est stigmatisé : «Gaz de schiste pour vous en forerplein les poches » / «bio gaz oui business gaz non» / "\$chi\$te». Le second argument des opposants refuse le gaz de schiste en tant qu'énergie. Sept slogans demandent de laisser le gaz « où il est », " reposer en paix». Deux jeunes enfants portant chacun une pancarte se répondent : «toc toc les messieurs [...] ${ }^{20}$, «Reste à ta maison, le gaz».

18. Voir figure 4 .

19. Nous soulignons.

20. Impossible de lire, sur la vidéo, la suite de ce slogan hapax. 
Peu d'arguments précis apparaissent : «si les gaz sortent de terre, ça promet l'enfer», "ne faisons pas sortir le mauvais génie de la terre». Cependant, cette menace fait référence à la préconisation du rapport annuel de l'Agence internationale de l'énergie ${ }^{21}$ de "laisser dans le sol plus des deux tiers des réserves prouvées de combustibles fossiles », afin de ne pas dépasser les $2{ }^{\circ} \mathrm{C}$ de réchauffement climatique d'ici la fin du siècle. La dimension globale de cet argument est caractéristique du discours de l'écologie politique.

Les pollutions générées par l'exploitation du gaz de schiste forment le noyau argumentatif de ce discours. La question de l'eau domine, et permet à la forme "gaz» de nouvelles actualisations, illustrées par ce chiasme : «Il y a de l'eau dans le gaz, il y a du gaz dans l'eau ». Pour les opposants, cette image renvoie au film Gasland de Josh Fox, lequel a selon F. Chateauraynaud (2011) «contribué à transformer l'exploitation des gaz de schiste en un nouveau front environnemental majeur du xxı ${ }^{\mathrm{e}}$ siècle». L'économiste Maxime Combes (2015, p. 85) explique que «la scène du film où Mike Markham enflamme son robinet d'eau avec un briquet en raison du gaz qu'il contient est beaucoup plus efficace que n'importe quel discours ou n'importe quelle étude». Plus largement, les informations sur les conséquences de l'exploitation du gaz de schiste aux États-Unis et au Canada ont joué un rôle déterminant dans la constitution d'un savoir citoyen sur cet objet.

La seconde pollution invoquée est visuelle, et constitue, nous l'avons déjà dit, un argument puissant. La citation poétique de Pierre Dac sur une affichette, "Il n’y a rien de plus difficile à consoler qu'un paysage désolé », souligne sa dimension esthétique.

\section{Les slogans identitaires}

Nous entrons, avec cette dimension, dans la partie la plus développée de notre corpus, partie que nous qualifions d'expressive et identitaire. Si l'on additionne les 7 slogans comprenant le nom «Ardèche », les 3 en occitan, et ceux comportant un déictique personnel de première personne, du singulier ou du pluriel, le total obtenu s'élève à 30 items. Au sein de cet ensemble, les mots «terre » (8 occurrences), « enfants», "santé» (3 occurrences chacun) se mêlent à l'expression du refus ou de l'inquiétude :

Pour nos enfants pour notre santé non aux gaz de schiste

Quel avenir pour nos enfants et notre terre?

Qu'allez vous faire de notre terre nourricière?

L'attachement à la «terre» qui s'exprime par le slogan capté «Touche pas à mon pote ${ }^{22}$, décliné en quatre versions, traduit l'élément central de ce discours identitaire :

21. Rapport publié le 12 novembre 2012 sous le titre World Energy Outlook 2012.

22. Créé par SOS Racisme en 1985, ce slogan est très connu et a été maintes fois détourné. 
Touche pas à mon schiste / à mon sous sol / à mon gaz / à la terre de mon papé

La menace de destruction est d'autant plus insupportable pour les riverains que le «sous-sol » évoqué appartient à l’État, et non au propriétaire du sol de surface, qui n'a par conséquent aucun intérêt à son exploitation. La délivrance par l'État de permis d'exploration à des compagnies pétrolières a donc été vécue comme une "agression », selon les témoignages de militants de collectifs ${ }^{23}$. La mise en scène de cette opposition se traduit formellement par la structure binaire «nous» vs "vous», typique de la façon de mobiliser l'énonciation dans les particitations de groupe (Maingueneau, 2012):

Tous vos dollars ne valent pas notre pinard

Non à vos profits oui à notre qualité de vie

Votre gaz bidon ne vaut pas notre picodon

Les éléments mis en avant, "pinard» et "picodon», pour anecdotiques qu'ils puissent paraitre, entrent en relation d'équivalence avec «qualité de vie ». Ils sont symboliques de choix auxquels les habitants (les néoruraux en particulier) tiennent. Pour mieux les appréhender, il faut envisager les slogans qui nomment ce territoire :

Ardèche Terre d'audace terre sans gaz

Gaz de schiste $=$ Ardèche martyrisée Ardèche à libérer

Ardèche en danger ardéchois cœur blessé

Protégeons notre Ardèche encore si belle

Le lien affectif, perceptible dans l'expression «notre Ardèche », s'exprime à travers la valorisation de la beauté de la région menacée, ainsi que par le rappel d'un passé douloureux, discrètement évoqué par les mots «martyrisée», " cœur blessé», "à libérer». Il s’agit ainsi de convoquer la dimension d’identité régionale et l'attachement à cette dernière.

Le Thésaurus dont l'hyperénonciateur fonde la compatibilité au sein de l'espace-temps énonciatif de la manifestation de Villeneuve-de-Berg révèle une logique qui nous parait dominée par le fonctionnement des slogans polyphoniques. Les slogans sources, captés et détournés par les nouvelles productions dans le but de connoter des situations historiques antérieures, entretiennent des relations. Nous avons étudié le réseau sémantique tissé entre «No pasarán », "Gardarem lo Larzac » et «No gazaran». L’oppresseur visé par le discours manifestant prend la figure de l'État, qui favorise, dans le cas du gaz de schiste, des entreprises multinationales dont l'objectif stigmatisé est le profit. Les personnages politiques qui le symbolisent sont raillés. À l'opposé, la communauté régionale, en soulignant les méfaits d'une possible exploitation, se regroupe autour de valeurs identitaires d'autant plus légitimes qu'elle

23. Témoignages rapportés en particulier dans le film No gazaran. 
n'a rien à gagner dans une entreprise exclusivement mercantile. Considérons à présent le second corpus de slogans, et l'évolution de ce Thésaurus.

\section{Cinq ans après, la manifestation de Barjac}

La seconde grande manifestation de la lutte contre le gaz de schiste se déroule à Barjac, le 28 février 2016. En effet, la loi du 13 juillet 2011 interdisant la fracturation hydraulique n'a qu'imparfaitement répondu aux problèmes soulevés par les opposants. En permettant, dans son article 2, la recherche ainsi que la possibilité d'autres techniques d'exploitation que celle interdite, elle « sauvegarde ce qui à long terme peut être considéré comme l'essentiel : les potentialités futures » (Baudrin et al., 2014). Les pétroliers qui ont reformulé leurs demandes de permis annulées en 2011 en recourant aux périphrases et autres reformulations ${ }^{24}$ pour ne plus mentionner le terme «fracturation » l'ont bien compris. C'est précisément la suppression de l'abrogation du permis de Montélimar, prononcée par le tribunal administratif de Cergy-Pontoise le 28 janvier 2016, qui a «mis en colère les gens», selon le maire de Barjac. La manifestation25 qui réunit un mois plus tard 71 collectifs d'opposition, 300 politiques locaux, des organisations locales et nationales (dont Attac 07, le Parti de Gauche ou EÉLV) a pour objectif l'abrogation définitive du permis de Montélimar. Est également réclamée une « loi interdisant toute exploration et exploitation d'hydrocarbures non conventionnels», promise par le gouvernement lors de la campagne électorale de 2012.

\section{Étude contrastive des slogans de Barjac}

Nous avons examiné ce second corpus de slogans dans une optique contrastive et synthétique. Les thématiques communes aux deux ensembles, qui présentent un nombre comparable de slogans en 2011 et 2016, portent sur le caractère politique général, la mention d'autres énergies, les profits des pétroliers, les slogans de refus («non à » / «stop à »). En revanche, certains thèmes développés en 2011 ont disparu en 2016 : les risques de pollutions (l'eau exceptée), la demande de laisser le gaz «là où il est », les forages. D’autres se voient fortement réduits, c'est le cas de la dimension identitaire. Le bloc thématique caractérisé par le déictique «nous» et les mots "santé», "terre», « enfants » passe de 17 unités à 7 . Les slogans revendiquant la dimension régionale (Ardèche, occitan) passent de 10 à 4. Des substitutions peuvent également être observées : en 2011, les critiques ciblées sur J.-L. Borloo et N. Sarkozy

24. Total parle ainsi de «stimulation de la roche-mère ».

25. Entre 7000 (préfecture) et 15000 (organisateurs) manifestants. 
totalisaient 17 items. Cinq ans après, c'est sur le pétrolier Total, bénéficiaire du permis restitué, que converge la contestation. Certains slogans datent de 2011: «Total'ment con / contre», d'autres fonctionnent selon les procédés déjà mentionnés : «Total dégaze d’ici et d'ailleurs », «n'inschistez pas». Des créations apparaissent, à l'image de ce poétique acrostiche :

\section{Terrorisme \\ Opaque \\ Toléré \\ A des fins \\ Liberticides!}

Les références contextuelles aux événements consécutifs à 2011, par exemple à la COP 21, constituent des différences évidentes. D’autres slogans expriment la durée de la lutte, tels «2011-2016 on est toujours là » " C'est toujours non », et l'entêtement des décideurs : "Le pouvoir vous rend sourd?", «Total dis-nous, dans “Non” c'est quoi que tu ne comprends pas?».

Les slogans de collectifs présentent les différences les plus significatives. Leur nombre se trouve multiplié par 4, et les objets des refus qu'ils expriment se multiplient. Au «gaz de schiste» (45 occurrences) viennent s'ajouter les huiles de schistes ${ }^{26}$ et le gaz de charbon 27 ( 3 occurrences chacun). Ces nouveaux objets concernent les autres régions menacées par les permis, le bassin parisien, le nord et l'est de la France. La présence des slogans de collectifs de ces régions indique la généralisation et l'extension nationale de la lutte. La dimension internationale se traduit, quant à elle, par des slogans en anglais et en allemand, dans lesquels le terme «frack» est central.

\section{Mother-Frackers}

Don't frack my mother28 ! Ni ici ni ailleurs

Au contraire, «fracturation» reste rare dans les slogans français. La raison tient selon nous au fait que la mobilisation s'est fondée en France, dès le début, sur une opposition à toute exploitation, quelle qu'en soit la technique. Au contraire, aux États-Unis, c'est le procédé de fracturation, massivement utilisé depuis les années 1990, qui focalise les revendications.

L'autre grande évolution des slogans de 2016 concerne la précision déictique «ni ici ni ailleurs, ni aujourd'hui ni demain ». Les opposants demandent une interdiction générale sur le plan spatial, et définitive sur le plan temporel. Ils appellent ainsi à corriger la loi Jacob (du nom du député UMP), à «étoffer cette loi insuffisante [...] pour une nouvelle loi qui cette fois ne permettra ni ruse

26. Ou pétrole non conventionnel.

27. Ou gaz de couche, qui s'exploite, contrairement au grisou, par fracturation.

28. Titre d'un clip du collectif «Artists against fracking». 
ni contournement ${ }^{29}$. En interdisant uniquement la fracturation hydraulique, la loi de 2011 avait interprété a minima les revendications. La présentation de cette loi comme une victoire de la contestation par la majorité des médias n'a pas empêché les collectifs de maintenir la mobilisation tout au long des cinq années suivantes. Comme l'exprime le collectif o7, cette mobilisation a «porté ses fruits», puisque la loi Hulot du 30 décembre 2017 «met fin à la recherche ainsi qu'à l'exploitation de tous les hydrocarbures liquides ou gazeux, quelle que soit la technique employée, à l'exception du gaz de mine »30.

Le discours manifestant étudié présente un réel intérêt comme matériau reflétant, tout en les construisant, les caractéristiques discursive et identitaire d'une controverse et de son évolution. Notre étude a montré qu'il refuse «hydrocarbures non conventionnels», le syntagme à visée scientifique des promoteurs, pour plébisciter la nomination "gaz de schiste ». Laquelle se charge alors des valeurs de la contestation puisque les mots, constate Paul Siblot, ont une mémoire qui «conserve l'empreinte de ceux qui, en les utilisant, y ont inscrit la posture qu'ils prenaient à l'égard de l'objet» (Siblot, 1999, p. 42). Les jeux permis par chacun des deux mots gaz et schiste participent de ce lestage sémantique: «gazar/er», «dégazer», "gazpiller», «kamigaz», «gazchis», pourl'un, « inschister», «masoschiste», « sarkoschiste», « fast-schiste » pour l'autre. Ils permettent également l'épanouissement de la fantaisie des énonciateurs et donnent à l'ethos de l'hyperénonciateur sa dimension humoristique. Le mot gaz autorise enfin les détournements d'un ensemble de slogans préexistants, qui dessinent un univers de références marqué par la lutte et la résistance. Qu'ils soient le produit d'énonciateurs collectifs ou individuels, ces slogans alimentent un Thésaurus dont les valeurs démocratiques dénoncent l'autocratie de certaines décisions de l’État. La circulation des slogans comme leur multiplicité (celle des slogans artisanaux surtout) expriment en effet l'horizontalité de l'organisation prônée par les collectifs. La convergence entre ces valeurs démocratiques et la revendication portée a renforcé la cohérence du mouvement, qui, en dépassant la simple communauté transitoire, a résisté pendant plus de cinq ans. Cette durée s'est montrée déterminante dans l'issue positive de la lutte. Cependant, les questions soulevées, relatives aux biens communs non substituables que sont l'eau, les paysages, et à la participation démocratique des citoyens aux décisions qui les concernent directement, restent posées, «ici [comme] ailleurs».

29. Allocution d’Édouard Chaulet, maire de Barjac.

30. Cette exception explique la poursuite de la mobilisation après la loi. 


\section{Références}

Baudrin Mathieu, Dauguet Benoît, Deias Dalia et al., 2014, " “On n'est pas des cowboys" : controverse sur l'exploitation des gaz de schiste et stratégie de l'industrie pétrolière », Revue d'anthropologie des connaissances, vol. VIII, nº 2, p.451-478.

CALVET Louis-Jean, 1976, La production révolutionnaire : slogans, affiches, chansons, Paris, Payot.

Chateauraynaud Francis, 2011, "L'affaire des gaz de schiste. Anatomie d'une mobilisation fulgurante ", Socio-informatique et argumentation, https://socioargu. hypotheses.org/3262 (consulté le 4 mars 2018).

COMBes Maxime, 2015, Sortons de l'âge des fossiles! : manifeste pour la transition, Paris, Seuil.

Fillieule Olivier, TARTAKowsky Danielle, 2014, La manifestation, Paris, Presses de Sciences Po.

GRINSHPUN Yana, 2013, "Discours manifestant et contestation universitaire (2009) », Argumentation et analyse du discours, http://journals.openedition.org/aad/1476 (consulté le 10 mars 2018).

GrUNIG Blanche-Noëlle, 1990, Les mots de la publicité : l'architecture du slogan, Paris, Presses du CNRS.

LAMBELET Alexandre, 2010, «Analyser les rassemblements au moyen de photographies ou de films. Pistes et enjeux», ethnographiques.org, http://www.ethnographiques. org/2010/Lambelet (consulté le 2 avril 2018).

LEROY Sarah, 2005, "Le détournement dans les titres de presse : un marquage dialogique? », dans Dialogisme, polyphonie : approches linguistiques, J. Bres, S. Mellet, P. P. Haillet et al. éd., Bruxelles, De Boeck-Duculot, p. 201-214.

LIBAERT Thierry, PIERLOT Jean-Marie, 2015, Les nouvelles luttes sociales et environnementales, Paris, Vuibert.

Maingueneau Dominique, 2016, Analyser les textes de communication, Paris, Armand Colin.

- 2012, Les phrases sans texte, Paris, Armand Colin.

- 2004, "Hyperénonciateur et “particitation” ", Langages, nº 156, p. 111-126.

Memmi Dominique, 1998, "Le corps protestataire aujourd'hui : une économie de la menace et de la présence », Sociétés contemporaines, n³1, p. 87-106.

Reboul Olivier, 1975, Le slogan, Bruxelles, Paris, Éditions Complexe, PUF.

Sıв Lot Paul, 1999, «De l'un à l'autre. Dialectique et dialogisme de la nomination identitaire », dans L'autre en discours, J. Bres, R. Delamotte-Legrand, F. Madray-Lesigne et al. éd., Montpellier, Université Paul-Valéry, p. 27-43.

TERRAL Pierre-Marie, 2017, Larzac terre de lutte : une contestation devenue référence, Toulouse, Privat.

Topçu Sezin, 2013, La France nucléaire : l'art de gouverner une technologie contestée, Paris, Seuil. 


\section{Résumé / Abstract / Compendio}

\section{« Non au GA(z de)CHIS(te)! " étude diachronique des slogans de manifesta- tions anti-gaz de schiste}

De nombreuses manifestations se sont déroulées en France entre 2011 et 2016 contre l'exploitation des gaz des schistes, autorisée par le gouvernement Sarkozy. À travers l'étude de deux corpus d'une centaine de slogans chacun, le présent article questionne ce discours manifestant. Que disent ces slogans des aspirations et valeurs de ce mouvement contestataire? Qui dénoncent-ils et sous quelles formes? Quels combats précédents convoquent-ils par la figure du détournement? L'examen de ces corpus originaux permettra de répondre à ces interrogations et de mettre en lumière la créativité d'un tel discours manifestant.

Mots-clés: gaz de schiste, discours manifestant, slogan, détournement de slogans, manifestation

\section{“Non au GA(z de)CHIS(te)!": a diachronic study of demonstration slogans against fracking}

Between 2011 and 2016, several demonstrations were set up against shale gas mining in France after it was authorized by the Sarkozy government. With two corpuses of about a hundred slogans each, the present article examines that demonstration discourse. What do the slogans tell of the aspirations and values of the protest movement? Who is being denounced and how? What previous fights do they refer to and repurpose? The study of two original corpuses will provide an answer to those questions while showing the inventiveness of that demonstration discourse.

Keywords: shale gas, demonstration discourse, slogan, repurposing slogans, demonstration

\section{«Non au GA(z de)CHIS(te) !»: estudio diacrónico de los eslóganes en las manifestaciones anti-gas de esquito}

Muchos manifestaciones ocurrieron en Francia entre el 2011 y el 2016 contra la explotación de los gases de esquito, autorizada por el gobierno Sarkozy. Basándose sobre el estudio de dos corpus de un centenar de eslóganes cada uno, este artículo cuestiona este discurso de la manifestación. ¿Quá dicen estos eslóganes relativamente a las aspiraciones y valores de este movimiento contestatario? ¿Qué denuncian y bajo qué formas? ¿Qué combates anteriores evocan en relación con la figura del desvío? El examen de estos corpus originales permitirá responder a estas preguntas y poner a la luz la creatividad de un tal discurso manifestante.

Palabras claves: gas de esquito, discurso manifestante, eslogan, desvío de eslóganes, manifestación 